\title{
Checklist of free living nematodes recorded from freshwater habitats in Southern Africa
}

\author{
J Heyns* \\ Department of Zoology, Rand Afrikaans University, PO Box 524, Auckland Park 2006, South Africa
}

\begin{abstract}
A checklist is given of about 150 species of aquatic and semi-aquatic free living nematodes recorded from freshwater as well as from mud and wet soil adjoining water bodies, from South Africa, Botswana and Namibia.
\end{abstract}

\section{Introduction}

The recently published key to genera of freshwater nematodes of Southern Africa (Heyns, 2002) did not list species, except in the case of a very few genera. A checklist is now presented for all freshwater species recorded from South Africa, Botswana and Namibia. It may serve as a supplement to the key. It should be noted, however, that while the key included all genera or higher taxa of which representatives have been recorded from freshwater, or semi-aquatic habitats often bordering on water bodies, the present checklist is limited to those species only which are known or assumed to be able to live and reproduce in aquatic or semiaquatic habitats, where they occupy a variety of niches in the ecosystem. At the same time it excludes those soil-living species, which are frequently washed into water bodies through the action of runoff rain or irrigation water. They end up in the benthos where they may survive for relatively long periods, but since they do not feed or reproduce here, they cannot play any significant role in the aquatic ecosystem (Heyns 1976; 2002). For this reason they are not included in the checklist, even though most publications on freshwater nematodes record these accidentals, probably for the sake of completeness. On the other hand there are some species, which can apparently live equally well in aquatic, semi-aquatic and terrestrial habitats. Where a particular publication lists such a species from more than one habitat, this is indicated in the checklist by using the following codes: aq (aquatic); s-aq (semi-aquatic) and $\mathrm{t}$ (terrestrial).

Synonyms are cited only when the species concerned have been recorded under names or genus-species combinations different from those currently in use. The number of the reference together with the code is then given after the name under which the species was recorded. Where the same species has been recorded more than once, but under different names or combinations, the relevant reference numbers and codes are given after each listing.

The classification followed in the checklist is a synthesis of those employed by Andrássy (1976; 1984 ) and Goodey (1963).

Order Rhabditida Oerley, 1880 (Chitwood, 1933)

Family Rhabditidae Oerley, 1880

Genus Rhabditis Dujardin, 1845

R. oxycerca (De Man, 1895) Dougherty, 1955

Genus Mesorhabditis Osche, 1952 (Dougherty, 1953)

M. striatica Dassonville and Heyns, 1984

Genus Diploscapter Cobb, 1913

D. coronata (Cobb, 1893) Cobb, 1913

Family Cephalobidae Filipjev, 1934

Genus Paracrobeles Heyns, 1968

P. laterellus Heyns, 1968

Dassonville (1981):

Dassonville \& Heyns (1984):

aq

Dassonville (1981):

aq

Rashid et al. (1990):

\footnotetext{
* Posthumously submitted by corresponding author: Prof. A AvenantOldewage, Rand Afrikaans University, PO Box 524, Auckland Park 2006, South Africa.

Im 011 489-2449; fax 011 489-2286; e-mail: ao@na.rau.ac.za

Received 5 February 2002; accepted in revised form 5 July 2002.
} 
Family Diplogasteridae Micoletzky, 1922

Genus Metadiplogaster Weingärtner, 1955 (Meyl, 1961)

M. secundus Dassonville and Heyns, 1984

Genus Paroigolaimella Paramonov, 1952

P. bernensis (Steiner, 1914) Andrássy, 1958

Dassonville \& Heyns (1984):

Dassonville \& Heyns (1984):

aq

Family Diplogasteroididae Filipjev and Schuurmans Stekhoven, 1941

Genus Rhabditidoides Rahm, 1928

R. stigmatus (Steiner, 1930) Andrássy, 1984

syn. Anchidiplogasteroides stigmatus (Steiner, 1930) Paramonov, 1964

Genus Goffartia Hirschmann, 1952

G. africana (Micoletzky, 1916) Hirschmann, 1952

syn. Diplogasteroides africanus Micoletzky, 1916

Family Diplogasteridae Micoletzky, 1922

Genus Diplogasteritus Paramonov, 1952

D. nudicapitatus (Steiner, 1914) Paramonov, 1952

syn. Acrostichus nudicapitatus (Steiner, 1914) Massey, 1962

Family Neodiplogasteridae Paramonov, 1952 (Andrássy, 1984)

Genus Mononchoides Rahm, 1928

M. aquaticus Dassonville and Heyns, 1984

M. changi Goodrich, Hechler and Taylor, 1968

M. gracilis

Family Teratocephalidae Andrássy, 1958

Genus Euteratocephalus Andrássy, 1958

E. capensis Heyns, 1977

E. palustris (De Man, 1880) Andrássy, 1958

E. spiraloides (Micoletzky, 1913) Heyns, 1977

Syn. Euteratocephalus hirschmannae Heyns, 1977
Dassonville \& Heyns (1984):

Micoletzky (1916):

Dassonville \& Heyns (1984):

Andrássy (1970):

Dassonville (1981):

Heyns (1977):

Swart et al (1991):
Dassonville \& Heyns (1984):

Dassonville \& Heyns (1984):

\section{Order Tylenchida Filipjev, 1934 (Thorne, 1949)}

Family Dolichodoridae Chitwood, 1950 (Skarbilovich, 1959)

Genus Neodolichodorus Andrássy, 1976

N. paralongicaudatus Rashid, Geraert and Heyns, 1990

Family Hoplolaimidae Filipjev, 1934 (Wieser, 1953)

Genus Helicotylenchus Steiner, 1945

H. africanus (Micoletzky, 1916) Andrássy, 1958

syn. Tylenchus africanus Micoletzky, 1916

Family Hemicycliophoridae Skarbilovich, 1959 (Geraert, 1966)

Genus Hemicycliophora De Man, 1921

H. labiata Colbran, 1960

H. lutosa Loof and Heyns, 1969

\begin{tabular}{|c|c|}
\hline Rashid et al. (1990): & s-aq \\
\hline $\begin{array}{l}\text { Botha \& Heyns (1993b): } \\
\text { Micoletzky (1916): }\end{array}$ & $\begin{array}{l}\mathrm{aq} \\
\mathrm{aq}\end{array}$ \\
\hline $\begin{array}{l}\text { Botha \& Heyns (1993b): } \\
\text { Loof \& Heyns (1969): } \\
\text { Loof \& Heyns (1969): }\end{array}$ & $\begin{array}{l}\text { aq; } \\
\text { s-aq } \\
\text { s-aq }\end{array}$ \\
\hline \\
\hline Dassonville \& Heyns (1984): & $\mathrm{aq}$ \\
\hline \multicolumn{2}{|l|}{ s Stekhoven, 1933} \\
\hline $\begin{array}{l}\text { Botha \& Heyns (1992c): } \\
\text { Heyns \& Coomans (1980): }\end{array}$ & $\begin{array}{l}\text { aq; } \\
\text { aq, t }\end{array}$ \\
\hline
\end{tabular}

Family Aphelenchoididae Skarbilovich, 1947 (Paramonov, 1953)

Genus Aphelenchoides Fischer, 1894

D. africanus Dassonville and Heyns, 1984

\section{Order Araeolaimida De Coninck and Schuurmans Stekhoven, 1933}

Family Leptolaimidae Oerley, 1880

Genus Chronogaster Cobb, 1913

C. africana Heyns and Coomans, 1980
Heyns \& Coomans (1980): 


\begin{tabular}{|c|c|c|}
\hline $\begin{array}{l}\text { C. glandifera Heyns and Coomans, } 1980 \\
\text { C. longicauda Heyns and Coomans, } 1980 \\
\text { C. multispinata Heyns and Coomans, } 1980\end{array}$ & $\begin{array}{l}\text { Dassonville (1981): } \\
\text { Heyns \& Coomans (1980): } \\
\text { Heyns \& Coomans (1980): } \\
\text { Heyns \& Coomans (1980): }\end{array}$ & $\begin{array}{l}\text { aq; } \\
\text { aq } \\
\text { aq } \\
\text { aq }\end{array}$ \\
\hline $\begin{array}{l}\text { Family Rhabdolaimidae Chitwood, } 1951 \\
\text { Genus Rhabdolaimus De Man, } 1880 \\
\quad \text { R. terrestris De Man, } 1880\end{array}$ & Botha \& Heyns (1993a): & $\mathrm{aq}$ \\
\hline $\begin{array}{l}\text { Family Plectidae Oerley, } \mathbf{1 8 8 0} \\
\text { Genus Plectus } \text { Bastian, } 1865 \\
\quad \text { P. cirratus } \text { Bastian, } 1865 \\
\text { P. sambesii Micoletzky, } 1916\end{array}$ & $\begin{array}{l}\text { Botha \& Heyns (1993b): } \\
\text { Micoletzky (1916): }\end{array}$ & $\mathrm{aq}$ \\
\hline \multicolumn{3}{|c|}{ Order Chromadorida Chitwood, 1933} \\
\hline $\begin{array}{l}\text { Family Cyatholaimidae Filipjev, } 1918 \\
\text { Genus Achromadora Cobb, 1913 } \\
\text { A. ruricola (De Man, 1880) Micoletzky, } 1925\end{array}$ & Botha \& Heyns (1992c): & aq \\
\hline $\begin{array}{l}\text { Family Desmodoridae Filipjev, } 1922 \\
\text { Genus Desmodora De Man, } 1889 \\
\quad \text { D. natalensis Swart and Heyns, } 1991\end{array}$ & Swart \& Heyns (1991b): & aq \\
\hline $\begin{array}{l}\text { Family Chromadoridae Filipjev, } 1917 \\
\text { Genus Chromadora } \text { Bastian, } 1865 \\
\text { C. monohystera } \text { Micoletzky, } 1916\end{array}$ & Micoletzky (1916): & aq \\
\hline \multicolumn{3}{|c|}{ Order Monhysterida Schuurmans Stekhoven and De Coninck, 1933} \\
\hline $\begin{array}{l}\text { Family Xyalidae Chitwood, } 1951 \\
\text { Genus Theristus Bastian, } 1865 \\
\quad \text { T. tessae Heyns and Coomans, } 1989\end{array}$ & Heyns \& Coomans (1989): & $\mathrm{aq}$ \\
\hline \multicolumn{3}{|l|}{ Family Monhysteridae De Man, 1876} \\
\hline \multicolumn{3}{|l|}{ Genus Monhystera Bastian, 1865} \\
\hline M. magnacephala Joubert and Heyns, 1980 & $\begin{array}{l}\text { Joubert \& Heyns (1980): } \\
\text { Jacobs et al. (1994): } \\
\text { Joubert \& Heyns (1980): }\end{array}$ & aq \\
\hline M. paludicola De Man, 1880 & Andrássy (1970): & $\mathrm{aq}$ \\
\hline M. taaiboschiensis Joubert and Heyns, 1980 & $\begin{array}{l}\text { Argo \& Heyns (1973): } \\
\text { Botha \& Heyns (1992c): } \\
\text { Dassonville (1981): } \\
\text { Joubert \& Heyns (1980): } \\
\text { Jacobs et al. (1994): } \\
\text { Joubert \& Heyns (1980): }\end{array}$ & $\begin{array}{l}\text { s-aq; } \\
\text { aq; } \\
\text { aq; } \\
\text { aq } \\
\text { aq; } \\
\text { aq }\end{array}$ \\
\hline $\begin{array}{l}\text { M. vulgaris De Man, } 1880 \\
\text { Genus Eumonhystera Andrássy, } 1981\end{array}$ & Micoletzky (1916): & $\mathrm{aq}$ \\
\hline E. filiformis (Bastian, 1865) & $\begin{array}{l}\text { Dassonville (1981): } \\
\text { Joubert \& Heyns (1980): }\end{array}$ & aq; \\
\hline $\begin{array}{l}\text { E. similis (Bütschli, 1873) Andrássy, } 1981 \\
\text { Genus Monhystrella Cobb, } 1918\end{array}$ & Micoletzky (1916): & $\mathrm{aq}$ \\
\hline $\begin{array}{l}\text { M. lepidura (Andrássy, 1963) Andrássy, } 1968 \\
\text { subspecies seelyae Heyns and Coomans, } 1990 \\
\text { M. paramacrura (Meyl, 1954) Andrássy, } 1968 \\
\text { M. parvella (Filipjev, 1931) Jacobs } 1987\end{array}$ & $\begin{array}{l}\text { Heyns \& Coomans (1990): } \\
\text { Heyns \& Coomans (1990); } \\
\text { Heyns \& Coomans (1990): }\end{array}$ & $\begin{array}{l}\mathrm{aq} \\
\mathrm{aq} \\
\mathrm{aq}\end{array}$ \\
\hline
\end{tabular}


Family Oncholaimidae Filipjev, 1918 (Baylis and Daubney, 1926)

Genus Oncholaimus Dujardin, 1845

O. deconincki Heyns and Coomans, 1977

O. jessicae Coomans and Heyns, 1986

Heyns \& Coomans (1977) :

Coomans \& Heyns (1986):

$\mathrm{aq}$

aq

Family Mononchulidae De Coninck, 1965

Genus Mononchulus Cobb, 1918

M. nodicaudatus (Daday, 1899) Cobb, 1918

Family Tripylidae Oerley, 1880

Genus Tripyla Bastian, 1865

T. glomerans Bastian, 1865

Genus Tobrilus Andrássy, 1959

T. ampiei (Joubert and Heyns, 1979)

T. diversipapillatus (Daday, 1905) Andrássy, 1959

T. elephas Andrássy, 1964

T. floridensis Joubert and Heyns, 1979

T. gracilis (Bastian, 1865) Andrássy, 1959

syn. Trilobus gracilis Bastian, 1865

T. longus (Leidy, 1852) Andrássy, 1959

T. stefanskii (Micoletzky, 1925) Andrássy, 1959

Genus Eutobrilus Tsalolikhin, 1981

E. annetteae (Joubert and Heyns, 1979) Tsalolikhin, 1981

syn. Tobrilus annetteae Joubert and Heyns, 1979

E. heptapapillatus (Joubert and Heyns, 1979) Tsalolikhin, 1981

syn. Trobrillus heptapapillatus Joubert and Heyns, 1979

Genus Tobriloides Loof, 1973

T. loofi Swart and Heyns, 1990

Family Prismatolaimidae Micoletzky, 1922

Genus Limonchulus Andrássy, 1963

L. heynsi Swart and Furstenberg, 1993

Genus Caprionchulus Swart and Heyns, 1993

C. diversipapillatus Swart and Heyns, 1993

Genus Onchulus Cobb, 1920

O. capriviensis Swart and Furstenberg, 1993

Family Ironidae De Man, 1876

Genus Ironus Bastian, 1865

I. crassatus Argo and Heyns, 1972

I. ernsti Argo and Heyns, 1972

I. ignavus Bastian, 1865

I. longicaudatus De Man, 1884

I. tenuicaudatus De Man, 1876

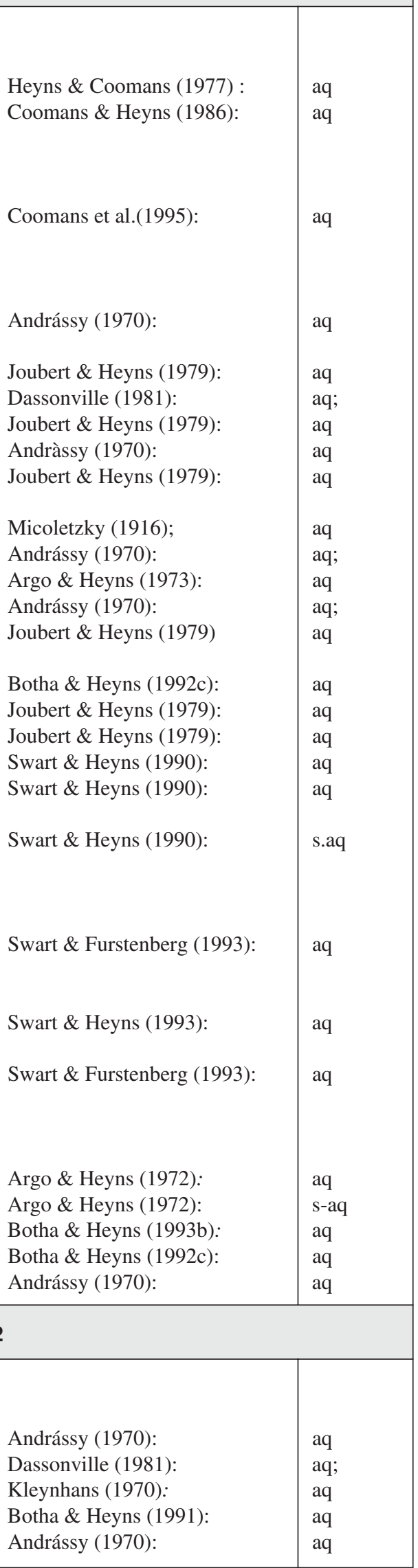

Order Dorylaimida Pearce, 1942

Family Dorylaimidae De Man, 1876

Genus Dorylaimus Dujardin, 1845

D. afghanicus Andrássy, 1960

D. asymphydorus Andrássy, 1969

D. deaconi Botha \& Heyns, 1991

D. pachys Andrássy, 1970

\begin{tabular}{|l|l|l|} 
& & \\
& & \\
& Andrássy (1970): & aq \\
Dassonville (1981): & aq; \\
& Kleynhans (1970): & aq \\
& Botha \& Heyns (1991): & aq \\
& Andrássy (1970): & aq \\
\hline
\end{tabular}


D. stagnalis Dujardin, 1845

D. stenus Andrássy, 1970

D. tepidus Andrássy, 1959

D. unicus Andrássy, 1970

Genus Ischiodorylaimus Andràssy, 1969

I. bathypyla Andrássy, 1970

I. gulliver (Andrássy, 1964) Andrássy, 1969

I. tessares Kleynhans, 1970

Genus Laimydorus Siddiqi, 1969

L. africanus Botha and Heyns, 1993

L. flavomaculatus (v. Linstow, 1876) Siddiqi, 1969

syn. Dorylaimus flavomaculatus v. Linstow, 1876

L. gazella Andrássy, 1970

L. olifanti Botha and Heyns, 1991

synonym of L. simplex (Baqri and Jana, 1983) Loof, 1996 according to Loof, 1996

Genus Namaquanema Heyns and Swart, 1993

N. hanki Heyns and Swart, 1993

Genus Mesodorylaimus Andrássy, 1959

M. aegypticus (Andrássy, 1958) Andrássy, 1959

M. arvensis (Cobb in Thorne and Swanger, 1936) Andrássy, 1959

M. bainsi Basson and Heyns, 1974

M. bastiani (Bütschli, 1873) Andrássy, 1959

syn. Dorylaimus bastiani Bütschli, 1873

M. importunus Basson and Heyns, 1974

M. intermedius Dassonville and Heyns, 1984

M. kowyni Basson and Heyns, 1974

M. mesonyctius (Kreis, 1930) Andrássy, 1959

M. paralitoralis Basson and Heyns, 1974

M. potus Heyns, 1963

M. pseudosubtilis Basson and Heyns, 1974

M. usitatus Basson and Heyns, 1974

M. vaalensis Heyns and Kruger, 1983

Possibly a synonym of M. alpestris (Thorne, 1939) Andrássy, 1959

Genus Drepanodorylaimus Jairajpuri, 1966

D. williamsi (Heyns and Kruger, 1983) Andrássy, 1986

syn. Mesodorylaimus williamsi Heyns and Kruger, 1983

Genus Prodorylaimus Andrássy, 1959

P. ensis Kleynhans, 1970

Genus Thornenema Andrássy, 1959

T. baldum (Thorne, 1939) Andrássy, 1959

Family Aporcelaimidae Heyns, 1965

Genus Aporcelaimellus Heyns, 1965

A. glandus Botha and Heyns, 1991

A. micropunctatus Botha and Heyns, 1990

A. obtusicaudatus (Bastian, 1865) Altherr, 1968

Family Nordiidae Jairajpuri and A.H. Siddiqi, 1964

Genus Lenonchium Siddiqi, 1965

L. fimbricaudatum Swart and Heyns, 1991

Family Actinolaimidae Thorne, 1939

Genus Neoactinolaimus Thorne, 1967

N. africanus (Filipjev, 1929) Thorne, 1967

syn. Actinolaimus africanus Filipjev, 1929
Micoletzky (1916):

Andrássy (1970):

Andrássy (1970):

Andrássy (1970):

Andrássy (1970):

Andrássy (1970):

Kleynhans (1970):

Botha \& Heyns (1993a):

Micoletzky (1916):

Andrássy (1970):

Botha \& Heyns (1991):

aq

$\mathrm{aq}$

Heyns \& Swart (1993):

Botha \& Heyns (1992a):

Dassonville (1981):

Basson \& Heyns (1975):

Heyns \& Kruger (1983):

Basson \& Heyns (1975):

Micoletzky (1916):

Basson \& Heyns (1975):

Dassonville \& Heyns (1984):

Basson \& Heyns (1975):

Basson \& Heyns (1975):

Heyns \& Kruger (1983):

Basson \& Heyns (1975):

Basson \& Heyns (1975):

Botha \& Heyns (1992a):

Heyns \& Kruger (1983)

Heyns \& Kruger (1983):

Basson \& Heyns (1975):

Heyns \& Kruger (1983):

Heyns \& Kruger (1983):

Heyns \& Kruger (1983):

aq

Kleynhans (1970):

Botha \& Heyns (1992a):

s-aq, t

s-aq

Botha \& Heyns (1991):

Botha \& Heyns (1991):

Andrássy (1970):

Swart \& Heyns (1991b):

Botha \& Heyns (1991):

Filipjev (1929): 
N. barbieri Vinciquerra and Heyns, 1984

N. brachydorus Vinciquerra and Heyns, 1984

N. crassidens Heyns and Argo, 1970

N. vaalensis Andrássy, 1970

Genus Mactinolaimus Andrássy, 1970

M. hutchinsoni (Filipjev, 1929) Thorne, 1967

syn. Actinolaimus hutchinsoni Filipjev, 1929

M. transkeiensis (Heyns and Argo, 1970) Vinciguerra, 1987

syn. Neoactinolaimus transkeiensis Heyns and Argo, 1970

Genus Paractinolaimus Meyl, 1957

P. macrolaimus (De Man, 1880) Andrássy, 1964

syn. Dorylaimus macrolaimus De Man, 1880

P. xosorum Heyns and Argo, 1970

Genus Afractinolaimus Andrássy, 1970

A. capensis (Heyns and Argo, 1970) Vinciguerra and Heyns, 1984

syn. Paractinolaimus capensis Heyns and Argo, 1970

A. magaliesmontanus (Heyns and Argo, 1970) Vinciquerra and Heyns, 1984 syn. Paractinolaimus magaliesmontanus Heyns and Argo, 1970

A. noblei Andrássy, 1970

A. zairensis (Baqri, Coomans and Van der Heiden, 1975) Vinciguerra and Heyns, 1984

Genus Actinca Andrássy, 1964

Family Carcharolaimidae Thorne, 1967

Genus Carcharolaimus Thorne, 1939

C. crassicostatus Heyns and Argo, 1970

Family Longidoridae Thorne, 1935

Genus Longidorus Micoletzky, 1922

L. heynsi Andrássy, 1970

Family Belondiridae Thorne, 1939

Genus Oxydirus Thorne, 1939

O. gangeticus Siddiqi, 1966

Genus Roqueus Thorne, 1964

R. africanus Andrássy, 1970

Family Leptonchidae Thorne, 1935

Genus Proleptonchus Lordello, 1955

P. krugeri Botha and Heyns, 1992

Family Mydonomidae Thorne, 1964

Genus Dorylaimoides Thorne and Swanger, 1936

D. longidens Furstenberg and Heyns, 1966

Family Aetholaimidae Jairajpuri, 1965

Genus Aetholaimus Williams, 1962

A. trochus Swart and Heyns, 1994

Family Alaimidae Micoletzky, 1992

Genus Alaimus De Man, 1880

A. primitivus De Man, 1880
A. intermedia Andrássy, 1968

\begin{tabular}{|c|c|}
\hline Vinciguerra \& Heyns (1984): & $\mathrm{aq}$ \\
\hline Vinciguerra \& Heyns (1984): & $\mathrm{aq}$ \\
\hline Heyns \& Argo (1970): & s-aq \\
\hline Andrássy (1970): & $\mathrm{aq}$ \\
\hline Filipjev (1929): & $\mathrm{aq}$ \\
\hline Heyns \& Argo (1970): & s-aq \\
\hline Micoletzky (1916): & $\mathrm{aq}$ \\
\hline Heyns \& Argo (1970): & $\mathrm{aq}$ \\
\hline Heyns \& Argo (1970): & s-aq \\
\hline Heyns \& Argo (1970): & s-aq \\
\hline Andrássy (1970): & $\mathrm{aq}$ \\
\hline Botha \& Heyns (1992b): & aq; \\
\hline Vinciguerra \& Heyns (1984): & $\mathrm{aq}$ \\
\hline Andrássy (1970): & $\mathrm{aq}$ \\
\hline Heyns \& Argo (1970): & s-aq \\
\hline Andrássy (1970): & $\mathrm{aq}$ \\
\hline Botha \& Heyns (1993a): & aq, s-aq \\
\hline Andrássy (1970): & $\mathrm{aq}$ \\
\hline Botha \& Heyns (1992b): & $\mathrm{aq}$ \\
\hline Furstenberg \& Heyns (1966): & $\mathrm{aq}$ \\
\hline Swart \& Heyns (1994): & $\mathrm{aq}$ \\
\hline Micoletzky (1916): & $\mathrm{aq}$ \\
\hline
\end{tabular}

Order Mononchida Jairajpuri, 1969 (Jairajpuri and Khan, 1982)

Family Mononchidae Filipjev, 1934

Genus Mononchus, Bastian, 1865

M. aquaticus Coetzee, 1968
Botha \& Heyns (1992c):

Coetzee (1968a): 


\section{M. tunbridgensis Bastian, 1865}

Genus Coomansus Jairajpuri and Khan, 1977

C. pretoriensis (Coetzee, 1968) Jairajpuri and Khan, 1977 syn. Mononchus pretoriensis Coetzee, 1968

Genus Clarkus Jairajpuri, 1970

C. sheri (Mulvey, 1967) Jairajpuri, 1970

syn. Mononchus jugalis Coetzee, 1968

Genus Iotonchus Cobb, 1916 (Pennak, 1953)

I. parabasidontus Mulvey and Jensen, 1967

I. risoceiae (Carvalho, 1955) Andrássy 1958

Genus Granonchulus Andrássy, 1958

G. subdecurrens Coetzee 1966

Genus Prionchulus Cobb, 1916 (Wu and Hoeppli, 1929)

P. muscorum (Dujardin, 1845) Wu and Hoeppli, 1929

Genus Cobbonchus Andrássy, 1958

C. artemisiae Coetzee, 1968

C. coetzeeae Andrássy, 1970

C. diannae Coetzee, 1965

C. megalus Coetzee, 1966

C. ockerti Coetzee, 1965

\section{Family Mylonchulidae Jairajpuri, 1969}

Genus Mylonchulus Cobb, 1916 (Altherr, 1953)

M. incurvus (Cobb, 1917) Andrássy, 1958

M. lacustris (Cobb N.A. in Cobb, M.V., 1915) Andrássy, 1958

M. minor (Cobb, 1893) Andrássy, 1958

M. montanus (Thorne, 1924) Andrássy, 1958

M. polonicus (Stefanski, 1915) Andrássy, 1958

[This may be a synonym of M. lacustris, see Coomans et al. (1995)]

\begin{tabular}{|l|l|} 
Coomans et al. (1995): & \\
Dassonville (1981): & $\mathrm{aq} ;$ \\
De Bruin \& Heyns (1992a): & $\mathrm{aq}, \mathrm{t}$ \\
Andrássy (1970): & $\mathrm{aq} ;$ \\
Botha \& Heyns (1992c): & $\mathrm{aq} ;$ \\
Coetzee (1968a): & $\mathrm{aq} ;$ \\
Coomans et al. (1995): & $\mathrm{aq;}$ \\
De Bruin \& Heyns (1992a): & $\mathrm{s}-\mathrm{aq}$ \\
Coetzee (1968b): & $\mathrm{aq}$ \\
& \\
Coetzee (1968a): & $\mathrm{aq}$ \\
& \\
Coetzee (1968a): & $\mathrm{s}-\mathrm{aq}, \mathrm{t}$ \\
De Bruin \& Heyns (1992b): & $\mathrm{s}-\mathrm{aq}$ \\
Coetzee (1967b) & $\mathrm{s}-\mathrm{aq} ;$ \\
Coomans et al. (1995): & $\mathrm{aq}$ \\
Coetzee (1966): & $\mathrm{s}-\mathrm{aq}, \mathrm{t}$ \\
Coetzee (1968a): & $\mathrm{s}-\mathrm{aq}, \mathrm{t}$ \\
Coetzee (1968b): & $\mathrm{s}-\mathrm{aq}$ \\
Andrássy (1970): & $\mathrm{aq}$ \\
Coetzee (1965): & $\mathrm{s}-\mathrm{aq}$ \\
Coetzee (1966): & $\mathrm{s}-\mathrm{aq}$ \\
Coetzee (1968b): & $\mathrm{s}-\mathrm{aq}$ \\
Coetzee (1968b): & $\mathrm{aq}$ \\
Botha \& Heyns (1992c): & $\mathrm{aq}$ \\
Coetzee (1967a) & \\
Andrássy (1970): & $\mathrm{aq}$ \\
& \\
& \\
&
\end{tabular}

\section{Acknowledgements}

Naomi Buckley of the Nematology Section of the ARC-PPRI is thanked for assistance with obtaining literature, and Susann Breytenbach of the Dept of Zoology, RAU, for typing the manuscript.

\section{Notification}

The manuscript was submitted posthumous by Annemariè AvenantOldewage (a former colleague) honouring the explicit request of the deceased author, as expressed to his wife shortly before he passed away.

\section{References}

ANDRÁSSY I (1970) Nematoden aus einigen Fluss-Systemen Südafrikas. Opusc. Zool. Budapest 10 179-219.

ANDRÁSSY I (1976) Evolution as a Basis for the Systematization of Nematodes. Pitman, London. 288 pp.

ANDRÁSSY I (1984) Klasse Nematoda. Gustav Fischer, Stuttgart. 509 pp.
ARGO A-D and HEYNS J (1972) Four new species of the genus Ironus Bastian, 1865 (Nematoda: Ironidae) from South Africa. Phytophylactica 4 59-66.

ARGO A-D and HEYNS J (1973) New and little known species of the nematode families Monhysteridae and Tripylidae from South Africa. Phytophylactica 5 149-154.

BASSON J B E and HEYNS J (1975) The genus Mesodorylaimus in South Africa (Nematoda: Dorylaimidae). Phytophylactica 6 (Year 1974) 249-260.

BOTHA A and HEYNS J (1991) Dorylaimoidea (Nematoda) from rivers in the Kruger National Park. Koedoe 34 1-24.

BOTHA A and HEYNS J (1992a) Freshwater nematodes of the genera Thornenema and Mesodorylaimus from the Kruger National Park with a diagnostic species compendium for South African species of the genus Mesodorylaimus (Nematoda: Dorylaimida). Koedoe 35 25-42.

BOTHA A and HEYNS J (1992b) Species of Tyleptus, Proleptonchus, Aquatides and Afractinolaimus from rivers in the Kruger National Park (Nematoda: Dorylaimida). Koedoe 35 43-54.

BOTHA A and HEYNS J (1992c) Further records and descriptions of nematodes from rivers in the Kruger National Park (orders Enoplida, Chromadorida, Monhysterida, Mononchida and Araeolaimida). Koedoe 35 11-25. 
BOTHA A and HEYNS J (1993a) Species of the genera Oxydirus, Dorylaimellus (Axodorylaimellus), Laimydorus and Rhabdolaimus from rivers in the Kruger National Park (Nematoda: Dorylaimida and Araeolaimida). Koedoe 36 49-60.

BOTHA A and HEYNS J (1993b) New records of Tylenchida, Araeolaimida and Enoplida from the Kruger National Park, with an addendum to the checklist of nematode species of the park. Koedoe 36 61-65.

COETZEE V (1965) South African species of the genus Cobbonchus Andrássy, 1958 (Nematoda: Mononchidae). Nematologica 11281 290.

COETZEE V (1966) Species of the genera Granonchulus and Cobbonchus (Mononchidae) occurring in southern Africa. Nematologica 12302 312.

COETZEE V (1967a) Species of the genus Mylonchulus (Nematoda: Mononchidae) occurring in southern Africa. Nematologica 12 (Year 1966) $557-567$.

COETZEE V (1967b) Species of the genus Iotonchus (Nematoda: Mononchidae) occurring in southern Africa. Nematologica 13367 377.

COETZEE V (1968a) Southern Africa species of the genera Mononchus and Prionchulus (Mononchidae). Nematologica 14 63-76.

COETZEE V (1968b) Mononchidae (Nematoda) of southern Africa. S. Afr. J. Agric. Sci. 11 403-414.

COOMANS A and HEYNS J (1986) Oncholaimusjessicaen. sp. (Nematoda: Oncholaimidae) from freshwater in the Transvaal. S. Afr. J. Zool. 21 197-201.

COOMANS A, RASHID F and HEYNS J (1995) On some predatory nematodes from the Okavango Delta, Botswana. Hydrobiol. 302 119131.

DASSONVILLE AF (1981) 'n Taksonomie en Ekologiese Studie van Varswater-nematode in Skinnerspruit, Pretoria. Unpublished Ph.D. Thesis, Rand Afrikaans University, Johannesburg. 222 pp.

DASSONVILLE AF and HEYNS J (1984) Freshwater nematodes from South Africa. 7. New and known species collected in Skinnerspruit, Pretoria. Phytophylactica 16 15-32.

DE BRUIN S and HEYNS J (1992a) Mononchida (Nematoda) of southern Africa: genera Mononchus Bastian, 1865, Clarkus Jairajpuri, 1970 and Coomansus Jairajpuri \& Khan, 1977. Phytophylactica 24 61-73.

DE BRUIN S and HEYNS J (1992b) Mononchida (Nematoda) from southern Africa: genus Iotonchus (Cobb, 1916) Altherr, 1950. Phytophylactica 24 39-60.

FILIPJEV IN (1929) Two new species of Actinolaimus from South Africa. Ann. Mag. Nat. Hist. London Serie 10 (4) 433-439.

FURSTENBERG JP and HEYNS J (1966) Chitwoodia transvaalensis n.gen., n.sp., and Dorylaimoides longidens n.sp., two new nematodes from South Africa. Proc. Helm. Soc. Wash. 33 26-30.

GOODEY T rewritten by GOODEY JB (1963) Soil and Freshwater Nematodes. Methuen, London. 544 pp.

HEYNS J (1976) Preliminary results of a survey of freshwater nematodes in South Africa. J. Limnol. Soc. South Afr. 2 43-45.

HEYNS J (1977) Freshwater nematodes from South Africa. 1. Euteratocephalus Andrássy, 1958. Nematologica 23 112-118.

HEYNS J (2002) Nematoda. In: Day JA and De Moor IJ (eds.) Guides to the Freshwater Invertebrates of Southern Africa, Vol. 5: NonArthropods. Water Research Commission, Pretoria.

HEYNS J and ARGO A-D (1970) Actinolaimoidea from South Africa (Nematoda: Dorylaimida). Phytophylactica 1 (Year 1969) 217-228.

HEYNS J and COOMANS A (1977) Freshwater nematodes from South Africa. 2. Oncholaimus deconincki n.sp. Rev. Zool. Afr. 91 906-912.

HEYNS J and COOMANS A (1980) Freshwater nematodes from South Africa 5. Chronogaster Cobb, 1913. Nematologica 26 187-208.

HEYNS J and COOMANS A (1989) A new freshwater species of Theristus from South West Africa/Namibia (Nematoda: Xyalidae). S. Afr. J. Zool. 24 95-98.
HEYNS J and COOMANS A (1990) Three Monhystrella species from inland waters in South West Africa - Namibia (Nematoda: Monhysteridae). Nematologica 35 (Year 1989) 1-14.

HEYNS J and KRUGER JC de W (1983) Freshwater nematodes from South Africa. 6. Mesodorylaimus Andrássy, 1959. Phytophylactica 15 47-53.

HEYNS J and SWART A (1993) Namaquanema hanki n.gen., n.sp. from South Africa (Nematoda: Dorylaimoidea). Fund. Appl. Nematol. 16 171-175.

JACOBS LJ, HEYNS J and COOMANS A (1994) Redescription of three South African Monhystera species (Nematoda). S. Afr. J. Zool. 29 62-69.

JOUBERT AP and HEYNS J (1979) Freshwater nematodes from South Africa. 3. Tobrilus Andrássy, 1959. J. Limnol. Soc. South. Afr. 5 $17-26$.

JOUBERT AP and HEYNS J (1980) Freshwater nematodes from South Africa. 4. The genus Monhystera Bastian, 1865. S. Afr. J. Zool. 15 190-197.

KLEYNHANS KPN (1970) New records and species of Dorylaimoidea (Nematoda) from South Africa. Phytophylactica 2 251-262.

LOOF PAA and HEYNS J (1969) Taxonomy of Hemicycliophora species from South Africa (Nematoda: Criconematoidea). Nematologica 15 464-472.

MICOLETZKY H (1916) Süsswasser - Nematoden aus Südafrika. Ergebnisse einer botanischen Forschungsreise nach Deutsch-Ostafrika und Südafrika (Kapland, Natal und Rhodesien). Denkschr. Kais. Akad. Wiss. Wien. 92 149-171.

RASHID F, GERAERT E and HEYNS J (1990) Neodolichodorus paralongi-caudatus sp.n. from Namibia (Nematoda: Tylenchida). Phytophylactica 22 281-283.

RASHID F, HEYNS J and COOMANS A (1990) Paracrobeles and Acrobeles species from South West Africa/Namibia with description of a new Acrobeles species (Nematoda: Cephalobidae). Phytophylactica 22 41-49.

SWART A andFURSTENBERG JP(1993) Description of two new species of the genera Onchulus and Limonchulus from Southern Africa (Nematoda: Enoplida, Onchulinae). Phytophylactica 25 11-19.

SWART A and HEYNS J (1988) Redescription of Eutobrilus heptapapillatus (Joubert and Heyns, 1979) Tsalolikhin, 1981 with notes on its morphology and a possible excretory system (Nematoda: Tobrilidae). Phytophylactica 20 161-168.

SWART A and HEYNS J (1990) Description of Tobriloides loofi n.sp. from Natal, South Africa (Nematoda: Onchulidae). S. Afr. J. Zool. 25 $138-143$.

SWART A and HEYNS J (1991a) Lenonchium frimbricaudatum n.sp. from South Africa, with a key to the species of Lenonchium (Nematoda: Nordiidae). Rev. Nematol. 14 413-418.

SWART A and HEYNS J (1991b) Desmodora (Sibayinema) natalensis subg. nov., spec. nov. from Lake Sibayi, South Africa (Nematoda: Desmodorida). Phytophylactica 23 273-278.

SWART A and HEYNS J (1993) Description of Caprionchulus diversipapillatus n.gen., n.sp. from Caprivi, Namibia (Nematoda: Onchulinae). S. Afr. J. Zool. 28 136-141.

SWART A and HEYNS J (1994) Description of Aetholaimus trochus n.sp. and the male of Ironus ignavus Bastian, 1865 (Nematoda) from Caprivi, Namibia. S. Afr. J. Zool. 29 54-61.

SWART A, DE WAELE D and HEYNS J (1991) A review of the genus Euteratocephalus Andrássy, 1958, with description of E. punctatus n.sp. Rev. Nematol. 14 551-563.

VINCIGUERRA MT and HEYNS J (1984) Freshwater nematodes from South Africa. 8. New and known species of Actinolaimoidea. S. Afr. J. Zool. 19 135-140. 